Revue internationale P.M.E.

Économie et gestion de la petite et moyenne entreprise

Revue

internationale

PME

\title{
Outils de gestion et espaces concurrentiels des PME
}

\section{Valérie Fernandez, Christian Picory et Frantz Rowe}

Volume 9, numéro 1, 1996

URI : https://id.erudit.org/iderudit/1008255ar

DOI : https://doi.org/10.7202/1008255ar

Aller au sommaire du numéro

\section{Éditeur(s)}

Presses de l’Université du Québec

ISSN

0776-5436 (imprimé)

1918-9699 (numérique)

Découvrir la revue

Citer cet article

Fernandez, V., Picory, C. \& Rowe, F. (1996). Outils de gestion et espaces concurrentiels des PME. Revue internationale P.M.E., 9(1), 79-102.

https://doi.org/10.7202/1008255ar

\section{Résumé de l'article}

À partir d'un traitement statistique, cet article analyse les dimensions instrumentales du contrôle de gestion (suivi, analyse et prévision), par rapport à des caractéristiques relevant à la fois de l'espace production et de l'espace marché, dont la conjonction définit l'espace concurrentiel. Dans cet esprit, l'étude proposera une caractérisation de la nature contingente du contrôle de gestion, d'une part, en fonction des contraintes qui pèsent sur les processus de production et, d'autre part, au moyen de l'étude des liens qui existent entre l'offre de la firme sur son marché, sa compétitivité et l'innovation technologique. Elle mettra ensuite en évidence un certain nombre d'affinités déterminantes pour l'étude de la contingence des outils de gestion en fonction de deux métamodèles de contrôle de gestion, dont les caractéristiques seront présentées. Ces analyses montreront que :

1) l'absence apparente de certains outils de gestion n'est pas rédhibitoire en soi et la croissance de l'entreprise ne peut y remédier que si la taille de l'entreprise est effectivement sous-optimale, par rapport à l'espace concurrentiel dans lequel elle s'inscrit;

2) l'orientation du contrôle ex post ou ex ante paraît liée à la conjonction de variables relevant à la fois de l'espace production et de l'espace marché. Cela permet de confirmer l'hypothèse selon laquelle l'analyse de la coordination et de l'organisation des activités de production s'inscrit dans une dialectique «firme-contexte de l'activité» et ce, en dépit du caractère extrêmement fluctuant de la frontière de la firme.
Ce document est protégé par la loi sur le droit d'auteur. L'utilisation des services d'Érudit (y compris la reproduction) est assujettie à sa politique d'utilisation que vous pouvez consulter en ligne.

https://apropos.erudit.org/fr/usagers/politique-dutilisation/ 


\title{
Outils de gestion et espaces concurrentiels des PME $^{1}$
}

\author{
Valérie FERNANDEZ \\ Christian PICORY \\ Frantz ROWE \\ École nationale supérieure \\ des télécommunications
}

MOTS CLÉS

\section{Espace concurrentiel-Outils de gestion Métamodèle de contrôle de gestion}

\begin{abstract}
RÉSUMÉ
$\grave{A}$ partir d'un traitement statistique, cet article analyse les dimensions instrumentales du contrôle de gestion (suivi, analyse et prévision), par rapport à des caractéristiques relevant à la fois de l'espace production et de l'espace marché, dont la conjonction définit l'espace concurrentiel. Dans cet esprit, l'étude proposera une caractérisation de la nature contingente du contrôle de gestion, d'une part, en fonction des contraintes qui pèsent sur les processus de production et, d'autre part, au moyen de l'étude des liens qui existent entre l'offre de la firme sur son marché, sa compétitivité et l'innovation technologique. Elle mettra ensuite en évidence un certain nombre d'affinités déterminantes pour l'étude de la contingence des outils de gestion en fonction de deux métamodèles de contrôle de gestion, dont les caractéristiques seront présentées. Ces analyses montreront que:
\end{abstract}

1. Ce travail s'inscrit dans le cadre d'une étude plus vaste réalisée au Département économie et management de l'ENST. L'enquête et l'analyse statistique ont été effectuées au département, avec l'aide de B. Geffroy ainsi que des précieux conseils de L. Lebart. Les interviews ont été réalisées par les étudiants de la promotion 1993. Que tous voient dans ces quelques mots l'expression de nos remerciements. 
1) l'absence apparente de certains outils de gestion n'est pas rédhibitoire en soi et la croissance de l'entreprise ne peut y remédier que si la taille de l'entreprise est effectivement sous-optimale, par rapport à l'espace concurrentiel dans lequel elle s'inscrit;

2) l'orientation du contrôle ex post ou ex ante paraît liée à la conjonction de variables relevant à la fois de l'espace production et de l'espace marché. Cela permet de confirmer l'hypothèse selon laquelle l'analyse de la coordination et de l'organisation des activités de production s'inscrit dans une dialectique «firme-contexte de l'activité" et ce, en dépit du caractère extrêmement fluctuant de la frontière de la firme.

\begin{abstract}
Based on a quantitative survey, this article analyzes the contingencies of the instrumental dimensions of management control (statistical reports, analysis tools, budgets and operational planning) within the framework of contingency theory. These contingencies belong both to production and to the market, the combination of which defines the "competitive space". The study analyzes the nature of management control, on the one hand by the production processes constraints and on the other hand by the relationships between the supply of the firm, its competitivity and technological innovation. Then a number of important issues for the study of management tools-given the existence of certain parameters affecting these relationships-are presented. Two management control metamodels can be distinguished. In addition, the analysis shows that:

1) The apparent lack of certain management tools is not always a major constraint. The growth of the firm will only lead to the creation of such tools if the size of the firm is under-optimal in its competitive space.

2) The ex post or ex ante orientation of control is linked both to characteristics of production and the market. This tends to confirm the hypothesis that the analysis of coordination and of the organization of production activities is placed in a dialectical "firm-activity context", even though the frontier of the firm greatly fluctuates.
\end{abstract}

\title{
RESUMEN
}

Este artículo analiza las dimensiones instrumentales del control de la gestión (seguimiento, análisis y previsión) a partir de un tratamiento estadístico en relación con las caracterísiticas propias al espacio de la producción y, al mismo tiempo, al espacio del mercado, cuya conjunción define el espacio competitivo. En este sentido, el estudio intentará, por una parte, caracterizar la naturaleza del control de la gestión en función de las dificultades que existen en el proceso de producción y, por otra parte, por medio del estudio de los lazos que existen entre la oferta de la firma sobre su mercado, su competitividad y la innovación tecnológica. Luego, mostrará con claridad un cierto número de afinidades determinantes para el estudio de la contigencia de los instrumentos 
de gestión en función de dos metamodelos de control de gestión cuyas caracterísiticas serán presentadas. Dicho análisis mostrará que:

1) La ausencia aparente de ciertos instrumentos de gestión no es un gran problema en sí y el crecimiento de la empresa sólo se puede solucionar si la talla de la empresa es realmente demasiado pequeña con relación a su espacio competitivo.

2) La orientación del control ex post o ex ante aparece unido a la conjunción de variables que conciernen, al mismo tiempo, el «espacio de producción» y el «espacio del mercado». Esto permite confirmar la hipótesis según la cual el análisis de la coordinación y de la organización de las actividades de producción se enmarcan dentro de la dialéctica "firma-contexto de la activida" y ello a pesar del carácter extremadamente fluctuante de la frontera de la firma.

\section{LES AUTEURS}

Valérie Fernandez est enseignante-chercheure à l'École nationale supérieure des télécommunications. Ses travaux portent sur l'analyse des organisations et, en particulier, sur la dynamique des modèles de comptabilité de gestion ; plusieurs études empiriques ont été réalisées sur ce thème dans le cadre notamment de contrats de recherche avec le CETIM, l'ANVAR, l'ANACT et le MRT.

Christian Picory est maître de conférences à l'École nationale supérieure des télécommunications (Paris-France). Domaines de spécialisation : histoire de la pensée économique, économie industrielle (organisation industrielle, financement, théorie de la firme). II est l'auteur de rapports d'études pour de grandes organisations publiques et privées ainsi que d'articles publiés dans Revue Économique, Revue d'Économie Industrielle, Revue Internationale PME... et consacrés au néoinstitutionnalisme, à l'analyse du financement de l'activité productive, à l'analyse des unités productives de petite dimension. II a également contribué à l'ouvrage Traité d'économie industrielle (Paris, Economica).

Frantz Rowe est professeur à l'Université de Nantes et à l'ENST. Ses travaux portent sur l'analyse des organisations et, en particulier, sur l'efficacité des télécommunications dans les entreprises. II a publié dans Sciences de Gestion, Revue Française de Gestion, Technological Forecasting and Social Change, ISDN Networks and Computer Systems, etc. et récemment "Des banques et des réseaux: productivité et avantages concurrentiels " (Economica, 1994). II est vice-président de l'Association Information et Management, et rédacteur en chef de la revue Systèmes d'Information et Management. Adresse : École nationale supérieure des télécommunications, Département économie et management, 46, rue Barrault, 75634 Paris, Cedex 13, France. 


\section{Introduction}

La distinction entre petite et grande entreprise constitue un point de passage obligé, qu'il s'agisse des analyses de la structuration de l'appareil productif ou encore de l'étude des pratiques de management des entreprises. Cette distinction s'est historiquement appuyée sur l'énoncé d'une "vérité » établie sur le plan théorique (Schumpeter, 1942) : la grande entreprise, et donc l'entreprise toujours plus grande, constituait la solution rationnelle du problème de l'organisation des activités productives. Cette «vérité » résultait d'un principe fondamental d'asymétrie entre les firmes : seules les entreprises de grande taille sont en mesure de consentir les investissements en capital risqué, nécessaires à la réduction de certains coûts et à l'innovation de produit qui, à son tour, soutient la croissance.

Cette thèse a cependant été battue en brèche à partir du début des années 1970. Les travaux de Schumacher (1973) sont tout d'abord venus réaffirmer l'importance des unités industrielles de petite dimension. Ensuite, l'observation a permis de reconsidérer une acceptation non critique du stéréotype de la grande dimension: aux États-Unis, scène naturelle de la marche triomphale vers la grande dimension, les événements ne se déroulaient en effet pas selon les prédictions. Non seulement les entreprises de petite dimension s'obstinaient à ne pas disparaître de l'industrie, mais encore, les effets de la tendance à la concentration de longue période ne se manifestaient pas avec une grande clarté. Enfin, les développements tardifs, liés à l'article séminal de Coase (1937) sur la nature de la firme sont venus remettre en question les fondements mêmes de la thèse sur l'efficacité comparée des firmes selon leur taille.

Cette évolution a conduit à la situation actuelle caractérisée, nous semble-t-il, par les trois propositions suivantes :

1. Il n'est plus possible d'admettre un rapport direct entre la dimension de la firme, quelle que soit la procédure de sa mesure (nombre de salariés, capitaux engagés, etc.), et sa performance économique.

2. La notion de taille de l'entreprise doit nécessairement intégrer le concept coasien de frontière de la firme qui permet de repérer l'unité de production dans son contexte et ce, en dépit du caractère intrinsèquement mobile de cette frontière.

3. Les analyses doivent tenir compte de l'extrême diversité des unités de petite dimension et, en particulier, de leurs besoins et de leur capacité de gestion.

Par rapport à ces propositions, la littérature consacrée aux outils de gestion des PME peut paraître singulièrement en retrait. Ainsi, en ce qui concerne l'analyse de la corrélation entre planification et performance, Robinson et 
Pearce (1984) ont montré dans leur étude que la planification stratégique existe dans certaines PME, mais elle est décrite comme incomplète, non structurée, irrégulière, sporadique, plutôt réactive et informelle. En outre, l'effet de la planification sur les performances est un sujet de controverse, puisqu'un effet positif reconnu par certains est remis en cause par d'autres. Pourtant, et ainsi que le notent d'Amboise et Bakanibona (1990), la littérature sur la planification pose deux difficultés principales.

Premièrement, la distinction entre planification stratégique et planification opérationnelle n'est pas toujours clairement effectuée, de sorte qu'il n'est pas toujours possible de différencier les objectifs, formalisés ou non, de long terme et le contrôle des activités de court terme. De notre point de vue, la réalité de la planification stratégique peut être mise en évidence par le couplage entre planification et analyse (de l'offre et de la demande). Parallèlement, la portée de la planification opérationnelle dépend de la définition d'indicateurs de performance et de leur suivi. Dans certains cas, il est possible de considérer qu'un pilotage «à vue», sans planification, est un mode de contrôle approprié à certains contextes.

Deuxièmement, beaucoup d'études effectuent une analyse basée sur des échantillons hétérogènes prélevés dans des secteurs d'activité différents, sans que l'effet de cette hétérogénéité sur les résultats soit contrôlé.

La prise en compte de la deuxième difficulté n'implique pas, nous semble-t-il, la restriction de l'analyse à des études monosectorielles qui interdisent tout rapprochement surtout lorsque les méthodologies sont par trop différentes. Elle suppose au contraire que l'on tienne compte de l'hétérogénéité du tissu des unités de petite dimension, qu'il s'agisse de l'appartenance sectorielle ou encore, et surtout, de l'environnement concurrentiel de la firme. En effet, le secteur d'activité d'appartenance constitue sans aucun doute un élément de différenciation important entre les divers groupes de PME. Pourtant, il ne saurait à lui seul, ou en croisement avec le critère de taille, rendre compte de la spécificité des PME ou structurer le tissu de ces entreprises. Comme suggéré plus haut, l'analyse doit intégrer la frontière de la firme, c'est-à-dire son positionnement dans un contexte particulier, au titre duquel il faut inclure les caractéristiques du processus de production (échelle, standardisation, production à la demande, production spécialisée, etc.), le niveau d'intensité technologique et de performance des processus innovants, les caractéristiques et le niveau d'expansion de la demande, le degré d'exposition à la concurrence (sur un marché national ou international), l'inscription de la firme dans un rapport de sous-traitance ou, encore, le soumissionnement aux marchés publics.

Le rôle de ces facteurs dans la structuration de l'appareil productif a fait l'objet de travaux effectués dans le cadre du SPRU (Science Policy Research Unit ) de l'Université du Sussex. Ces travaux ont permis de proposer une 
nouvelle nomenclature de secteurs industriels en liaison avec les caractéristiques précédentes et, en particulier, avec les processus d'innovation technologique (Dosi, Pavitt et Soete, 1985 ; Pavitt 1984). En partant de ces caractéristiques et en reformulant, sur cette base, la notion de secteur que nous avons appelé espace concurrentiel, il a été possible de définir une typologie de PME qui distingue les PME traditionnelles, les PME caractérisées par une dimension organisationnelle élevée (scale-intensive), les PME orientées par la demande (specializedsuppliers) et les PME caractérisées par une intensité élevée de recherche de base (science-based). Cette typologie permet une analyse plus fine de la dynamique de ces firmes et de leurs stratégies (Picory, 1990 a et b). Dans le cadre de cette approche, la contribution propose d'analyser les outils de gestion (suivi, analyse et prévision ${ }^{2}$ ) des PME. L'hypothèse fondamentale de ce travail est que le contrôle de gestion qui s'opère dans les organisations de petite taille à partir d'outils de gestion est contingent au contexte socio-économique dans lequel s'inscrit la PME. Autrement dit, il existerait une relation entre la nature de l'espace concurrentiel (défini en fonction de configurations particulières des caractéristiques évoquées plus haut) auquel appartient la PME et la nature, l'ampleur et l'intensité du suivi, de l'analyse et de la prévision de l'activité.

L'examen de cette hypothèse a été effectué à partir du traitement statistique des données obtenues sur un échantillon de 102 petites et moyennes entreprises (dont le nombre de salariés est inférieur ou égal à 500) appartenant à des secteurs d'activité essentiellement industriels et situées dans la région Île-deFrance $^{3}$. L'analyse proposera dans une première section une identification des différents espaces concurrentiels dans lesquels s'inscrivent les PME, afin de cerner la contingence des outils de gestion au moyen des contraintes relevant non seulement des technologies de production mises en œuvre (échelle, gamme, variété, innovation), qui renvoient principalement à l'espace production, mais encore, des propriétés de l'offre et de la demande du marché, lesquelles renvoient essentiellement à l'espace marché.

2. Notre discrimination des outils de gestion (Fernandez, Picory et Rowe, 1994) s'inscrit dans la perspective des travaux d'Anthony (1988) où le contrôle de gestion (au sens anglo-saxon du terme) est défini, dans sa dimension instrumentale, comme un processus en trois phases : planification, pilotage et évaluation. Nous avons ainsi associé à chaque phase du processus de contrôle, une batterie d'outils de gestion : outils de suivi correspondant à la phase « pilotage », outils d'analyse correspondant à la phase «évaluation » et outils de prévision correspondant à la phase «planification ».

3. La méthodologie de cette enquête ainsi que les caractéristiques de l'échantillon sont présentées dans Fernandez, Picory et Rowe (1993). Sur la représentativité de notre échantillon, on consultera l'annexe II qui présente un tableau comparatif entre les statistiques du SESSI (1990/ 1991), l'échantillon de la Centrale des Bilans de la Banque de France et l'échantillon constitué pour la présente étude (PME industrielles). 
Cette analyse permettra alors de mettre en évidence un certain nombre d'affinités déterminantes pour l'étude de la contingence des outils de gestion en fonction de deux métamodèles de contrôle de gestion, dont les caractéristiques seront présentées dans la deuxième section de cet article.

\section{Technologie, caractéristiques de l'offre et de la demande et outils de gestion}

L'espace concurrentiel dans lequel s'inscrit la PME peut être identifié à partir de cinq axes d'analyse.

1. Le premier concerne l'asymétrie technologique entre les secteurs. Il s'agit dans ce cas de tenir compte de l'importance des innovations radicales (science-based) par opposition aux innovations incrémentales le long d'une trajectoire technologique, des barrières à l'entrée liées à l'appropriabilité des connaissances, de la rapidité de la diffusion, des avantages associés à l'innovation, de l'importance, enfin, de l'utilisateur (intermédiaire ou final) dans les processus d'innovation.

2. Le deuxième prend en compte l'interdépendance technologique des entreprises. Il s'agit alors d'analyser le processus de diffusion de l'innovation entre firmes, que ce soit à l'intérieur d'un secteur ou entre secteurs différents et, par la suite, de définir autant que faire se peut les liens entre secteurs générateurs nets d'innovations technologiques et secteurs utilisateurs nets de technologie.

3. Le troisième renvoie aux économies d'échelle liées non seulement aux innovations procédés, mais aussi aux innovations non technologiques (organisation, gestion).

4. Le quatrième concerne l'investissement «innovant » et les formes de transfert de technologie (cession de licences, brevets, assistance technique, accords de spécialisation productives, etc.). Il s'agit dans ce cas d'examiner l'influence de ce facteur sur la structuration du système productif.

5. Le cinquième est lié à la dimension des entreprises. Il s'agit d'étudier le rôle de l'effet de seuil non seulement technologique, mais aussi financier dans les processus d'innovation en liaison, en particulier, avec la nature de la spécialisation des firmes sur des produits particuliers (standardisés ou différenciés...).

À partir de ces cinq axes d'analyse, les travaux du SPRU (Science Policy Research Unit) de l'Université du Sussex identifient quatre secteurs industriels ${ }^{4}$.

4. Sur la composition de ces secteurs, on consultera l'annexe 1. 
1. Les secteurs caractérisés par une intensité élevée de recherche de base (science based). Nous les noterons $S B$ pour la suite du texte. Ces secteurs sont généralement générateurs nets d'innovation technologique. Ils sont caractérisés par une forte activité de recherche de base soit au niveau de l'entreprise, soit au niveau de structures plus larges telles que les technopoles, par une lente "gestation» des projets innovants opposée à un cycle de vie des produits très rapide, par une très forte dépendance par rapport aux structures de recherche publiques ou privées, et, enfin, par une faible sensibilité par rapport aux facteurs de compétitivité prix. Des exemples classiques peuvent être trouvés parmi les industries des télécommunications, des composants actifs, de l'aérospatiale, de l'informatique, des nouveaux matériaux de synthèse ou des diverses applications des biotechnologies.

2. Les secteurs caractérisés par une dimension organisationnelle élevée (scale intensive). Nous les noterons SI pour la suite du texte. Les caractéristiques de ces secteurs sont les suivantes: des productions à haut volume et une grande flexibilité de gamme, une intense activité de recherche appliquée aux innovations procédés, une forte propension à incorporer des innovations produits issues d'autres secteurs, une dynamique de l'innovation de procédés ou de l'organisation contrainte par une grande sensibilité aux coûts des inputs (énergie, travail, matières premières) ainsi qu'à la concurrence sur les prix relativement plus élevée, de fortes dépenses en liaison avec la publicité, le marketing, les services après-vente. Les industries de l'automobile, de l'électroménager «blanc», ainsi qu'une large part des industries de la pharmacie constituent quelques exemples de secteurs scale intensive.

3. Les secteurs orientés par la demande (specialized suppliers). Nous les noterons $S S$ pour la suite du texte. Ceux-ci sont caractérisés par une grande flexibilité de leur production par rapport aux exigences spécifiques de leurs clients, qu'il s'agisse d'utilisateurs intermédiaires ou finaux, par une prévalence des entreprises de taille relativement limitée, par une spécialisation élevée sur des productions particulières ou dans le cadre de «niches » technologiques restreintes, par une très forte concurrence hors prix (non price competition) sur la flexibilité, par la fiabilité et la qualité des produits, par les capacités d'adaptation et d'assistance. Des exemples aujourd'hui classiques peuvent être trouvés parmi les industries de la mécanique de précision, des instruments de mesure, de l'appareillage biomédical, etc.

4. Les secteurs traditionnels, notés ST. Ces derniers sont utilisateurs nets d'innovations produits et procédés développées dans les autres secteurs. 
Ils mobilisent relativement peu de moyens en faveur du poste R-D. Comme pour les secteurs caractérisés par une dimension organisationnelle élevée $S I$, les innovations procédés s'inscrivent dans le cadre d'une stratégie de réduction des coûts. L'environnement concurrentiel est non seulement déterminé par les facteurs prix, mais aussi par des facteurs hors prix tels que le design, la qualité, la fiabilité, la variété de gamme, la compétence des services marketing et des réseaux de distribution commerciale. En exemple, nous citerons les industries de l'habillement (prêt-à-porter, chaussure, textile, cuirs et peaux, etc.), de l'ameublement, du jouet et des instruments de musique, etc.

Cette typologie transversale, aux taxonomies usuelles, est fondée sur des caractéristiques de l'offre et de la demande qui conduisent à une composition de secteurs extrêmement diversifiée si nous ne retenons que le concept classique de produit homogène. Il suffit de reprendre, pour chacun d'entre eux, la liste des exemples pour s'en convaincre. Elle constitue cependant un outil permettant de saisir la place des PME dans la structure de l'appareil productif et, par la suite, de comprendre leur rôle dans la structuration de l'offre nationale.

Sur la base des indications qui précèdent, nous avons alors regroupé les 102 PME industrielles de l'échantillon en fonction, d'une part, de l'activité supposée homogène et repérée par le code $\mathrm{APE}^{5}$ et, d'autre part, de la table de correspondance (annexe I) entre ces activités et les secteurs au sens de Pavitt (1984).

L'exploitation des données obtenues dans le cadre de cette approche nous permet alors d'obtenir les résultats synthétisés dans le tableau 1 de la page suivante ${ }^{6}$.

5. Rappelons que, sur la base d'une description de l'activité de la PME, les codes APE ont été recodés.

6. Nous ne retiendrons, dans toutes les caractérisations qui suivent, que des différences entre le pourcentage des firmes d'une classe vérifiant une modalité donnée et le même pourcentage mais, sur l'ensemble de l'échantillon, substantiellement significative. Autrement dit, nous ne nous fonderons, en règle générale, que sur des écarts entre la première et la seconde grandeur, tels que la probabilité pour que cet écart soit dû au hasard est inférieure à $5 \%$, ce qui correspond à une valeur test supérieure ou égale à 1,66 en valeur absolue. Lorsque la valeur test est positive, la modalité est caractéristique des individus de la classe ; dans le cas contraire, elle ne saurait les caractériser. Pour de rares cas, des valeurs tests légèrement inférieures seront mentionnées, mais uniquement lorsque les modalités, auxquelles elles se réfèrent, confirment des corrélations statistiques établies selon la règle précédente. Dans les développements qui suivent ne seront indiquées que les valeurs tests. 
Tableau 1

Secteurs au sens de Pavitt et outils de gestion

\begin{tabular}{|c|c|c|c|}
\hline $\begin{array}{l}\text { SECTEURS (effectifs) } \\
\text { Effectif total = 102 }\end{array}$ & $\begin{array}{l}\text { Indicateur } \\
\text { de suivi }\end{array}$ & $\begin{array}{c}\text { Indicateur } \\
\text { d'analyse }\end{array}$ & $\begin{array}{l}\text { Indicateur } \\
\text { de prévision }\end{array}$ \\
\hline TRADITIONNELS (21) & $\begin{array}{c}\text { Supérieur } \\
1,96\end{array}$ & $\begin{array}{c}\text { Normal } \\
\mathbf{1 , 2 8}\end{array}$ & \\
\hline \multicolumn{4}{|l|}{ Production non standardisée $(2,95)$} \\
\hline Gamme de produits étendue $(2,13)$ & Faible & & \\
\hline $\begin{array}{l}\text { Production différenciée selon exigences } \\
\text { de la clientèle }(1,83)\end{array}$ & $-1,73$ & & \\
\hline \multicolumn{4}{|l|}{$\begin{array}{l}\text { Production non conditionnée par les choix } \\
\text { d'innovation }(1,56)\end{array}$} \\
\hline \multicolumn{4}{|l|}{$\begin{array}{l}\text { Très forte concurrence sur les coûts des } \\
\text { produits }(1,60)\end{array}$} \\
\hline \multicolumn{4}{|l|}{25 à $49 \%$ de sous-traitance dans le CA $(1,51)$} \\
\hline SCALE INTENSIVE (11) & $\begin{array}{c}\text { Très faible } \\
2,14 \\
\end{array}$ & & $\begin{array}{c}\text { Normal } \\
\mathbf{2 , 2 1}\end{array}$ \\
\hline
\end{tabular}

Production sur grandes séries $(4,57)$

Production standardisée $(2,91)$

Gamme de produits étendue $(1,71)$

Production contrainte par les économies d'échelle $(1,54)$

Plus de 500 salariés $(2,66)$

$\mathrm{CA}>500 \mathrm{MF}(2,33)$

Exportation $(1,94)$

\begin{tabular}{|c|c|c|}
\hline SPECIALIZED SUPPLIERS (60) & $\begin{array}{c}\text { Normal } \\
-1,65 \\
\end{array}$ & $\begin{array}{c}\text { Très inférieur } \\
1,6 \\
\end{array}$ \\
\hline $\begin{array}{l}\text { Pas de grandes séries }(6,22) \\
\text { Pas de contrainte de rech. fondamentale ou } \\
\text { appliquée }(3,99) \\
\text { Production différenciée selon exigences } \\
\quad \text { de la clientèle }(3,63) \\
\text { Production contrainte par les facteurs } \\
\quad \text { de compétitivité prix }(3,39) \\
\text { Pas de gamme de produits étendue }(3,30) \\
\text { Pas de contrainte d'innovation et de haut } \\
\text { contenu technologique }(2,50) \\
\text { Activité non orientée par les efforts de } \\
\quad \text { publicité et d'emballage }(2,37) \\
\text { Pas de contrainte d'économies d'échelle }(1,90) \\
\text { Entreprises autonomes }(4,62) \\
\text { CA inférieur à } 50 \text { MF }(1,76) \\
10 \text { à } 19 \text { donneurs d'ordre }(1,63) \\
1 \text { à } 9 \text { salariés }(1,50)\end{array}$ & & $\begin{array}{l}\text { Normal } \\
-2,56\end{array}$ \\
\hline SCIENCE BASED (10) & & $\begin{array}{l}\text { Normal } \\
2,66\end{array}$ \\
\hline $\begin{array}{l}\text { Production contrainte par la qualité de la } \\
\text { recherche fondamentale }(3,78)\end{array}$ & & \\
\hline $\begin{array}{l}\text { Production conditionnée par les choix } \\
\text { innovation et haut contenu } \\
\text { technologique }(3,67)\end{array}$ & & $\begin{array}{l}\text { Inférieur } \\
-1,56\end{array}$ \\
\hline $\begin{array}{l}\text { Gamme de produits étendue }(2,37) \\
\text { Production sur grandes séries }(2,16) \\
\text { Plus de } 500 \text { salariés }(2,83) \\
\mathrm{CA}>500 \mathrm{MF}(2,52) \\
\text { Export : } 30 \text { à } 49 \% \text { du CA }(1,96) \\
\text { Export : } 75 \text { à } 100 \% \text { du CA }(1,85) \\
\text { Marchés publics } 25 \text { à } 49 \% \text { du CA }(1,58)\end{array}$ & & \\
\hline
\end{tabular}


Remarquons tout d'abord que les secteurs construits a priori sur la base des codes APE vérifient bien, pour des seuils statistiques très significatifs, les caractéristiques des secteurs au sens de Pavitt (1984). En outre, ces résultats nous permettent de caractériser le degré de développement des outils de gestion des PME appartenant à chacun de ces secteurs. Ainsi, à partir de trois batteries de questions concernant le suivi, l'analyse et la prévision, nous avons procédé à la construction d'autant de macrovariables. Leur valeur constitue un indicateur de l'intensité du suivi, de l'analyse ou de la prévision ${ }^{7}$. Les caractéristiques des différents outils de gestion peuvent être résumées sous la forme de quatre propositions.

\section{Proposition 1: Les PME des secteurs traditionnels (ST) sont plutôt carac- térisées par des outils de suivi supérieurement développés et des outils d'analyse normalement développés.}

Pour les seuils statistiques retenus, le suivi concerne, exclusivement la production. Suivi du taux d'utilisation des équipements : une fois par semaine $(2,16)$, suivi du taux de rotation des stocks de matière : une fois par mois $(1,73)$, du taux de production retouchée ou rebutée : une fois par semaine $(1,51)$, et des écarts sur prévisions des quantités : une fois par mois $(1,35)$.

En matière d'analyse, la caractéristique la plus marquante est l'adaptation de l'offre aux caractéristiques de la clientèle au moyen du choix du réseau de distribution $(1,79)$. Cette caractéristique renvoie alors plutôt à une

7. Pour les questions sur le suivi, plus la fréquence du suivi est élevée, plus l'entreprise capitalise de points pour chacune des 14 questions (de 0 pour jamais à 5 pour un suivi journalier). Le score d'une entreprise s'échelonne donc de 0 à 70 après sommation des questions sur le suivi. L'intensité de la prévision est évaluée d'après 6 questions bimodales (oui / non). Pour chacune d'elles, l'entreprise capitalise un point pour une réponse positive, aucun pour une réponse négative. Après sommation des résultats aux 6 questions, la valeur de l'intensité de la prévision pour une entreprise s'échelonne entre 0 et 6.

Enfin, l'intensité de l'analyse est évaluée suivant le même principe que pour l'indicateur précédent. Toutefois, les deux derniers groupes de questions (« Existe-t-il une politique de segmentation de votre clientèle par... ? » et «Quelle utilisation faites-vous de vos calculs de coûts ? ») font l'objet d'une pondération double. La question portant sur les modalités de calcul du coût de revient a été traitée en accordant trois points pour la première modalité, deux pour la seconde et enfin un seul pour la troisième.

La cohérence interne de chacune des macrovariables a été testée en calculant l'indice de Cronbach :

Cronbach-alpha $=0,73$ pour les macrovariables de suivi et d'analyse ;

Cronbach-alpha $=0,65$ pour la macrovariable de prévision.

Ces résultats indiquent une construction satisfaisante des macrovariables. 
stratégie de réduction des coûts et d'amélioration de la qualité, en raison en particulier de l'environnement concurrentiel, déterminé non seulement par les facteurs prix, mais encore par des facteurs hors prix tels que le design, la qualité, la fiabilité, la variété de gamme, la compétence des réseaux de distribution commerciale. Enfin, compte tenu des seuils statistiques retenus, les caractéristiques des outils de prévision n'apparaissent pas significativement ${ }^{8}$.

Proposition 2: Les PME des secteurs de dimension organisationnelle élevée (SI) sont plutôt caractérisées par un indicateur de suivi très faible et un indicateur de prévision normal.

Le suivi porte sur les éléments suivants : sur la production, avec le suivi des écarts sur les prévisions des quantités $(1,51)$, sur le commercial, avec le suivi des délais fournisseurs une fois par an $(1,57)$ et celui des délais clients une fois par trimestre $(1,54)$, sur la finance, enfin, avec le suivi de la marge commerciale une fois par mois $(1,76)$. En matière d'analyse, il est possible d'observer une adaptation de l'offre aux caractéristiques de la clientèle par le choix du réseau de distribution $(2,26)$, une vérification systématique de la solvabilité du nouveau client $(1,82)$, en général, par l'intermédiaire d'un organisme spécialisé $(1,72)$. La prévision, enfin, comporte un plan prévisionnel de production $(2,34)$, un plan prévisionnel des ventes $(1,86)$ ainsi qu'une analyse de la rentabilité prévisionnelle des investissements.

Clairement, les caractéristiques qui viennent d'être décrites renvoient massivement à l'échelle de la production.

Proposition $3:$ Les PME des secteurs orientés par la demande (SS) sont plutôt caractérisées par un indicateur de prévision très inférieur.

Si le suivi est absent de cette caractérisation par l'intermédiaire de son indicateur synthétique, il est cependant possible de donner quelques indications. S'agissant de la production, l'attention est portée sur le suivi du coût unitaire de fabrication $(2,53)$. Par contre, ne font jamais l'objet d'une attention particulière le suivi du nombre d'heures travaillées par rapport au nombre d'heures ouvrées $(2,03)$, le suivi des écarts sur prévision des quantités $(1,94)$ et celui du taux d'utilisation des équipements $(1,70)$. Le suivi commercial est entièrement orienté vers la clientèle, puisqu'il comporte le suivi du taux de satisfaction de la clientèle une fois par jour $(1,87)$ et celui des délais clientèle une fois par jour également $(1,81)$, à l'exclusion cependant de celui du nombre de commandes $(1,78)$. Ces observations sont naturellement liées, d'une part,

8. Ce résultat tend à corroborer les conclusions d'Anthony (1988) quant à l'influence d'un environnement externe « prévisible » sur l'absence de programmation. 
à la grande flexibilité de l'appareil de production en fonction des exigences spécifiques de la clientèle et, d'autre part, à l'existence de relations de soustraitance stables ou suffisamment nombreuses qui permettent de dispenser la PME de suivre le nombre de commandes. La fréquence du suivi financier est mensuelle, probablement en raison des problèmes de trésorerie liés à la relation de sous-traitance. Ce suivi financier comporte le suivi mensuel de la trésorerie $(1,98)$ et celui du chiffre d'affaires $(1,84)$. Le suivi de la marge commerciale fait l'objet d'une attention moins serrée, puisqu'elle est contrôlée une fois par trimestre.

Les performances de l'indicateur de prévision renvoient, quant à elles, à l'inexistence de l'estimation de la rentabilité prévisionnelle des investissements $(2,37)$, d'un plan pluriannuel d'investissement $(1,60)$ ou d'un plan prévisionnel des ventes $(1,45)$.

Proposition 4: Les PME secteurs à intensité élevée de recherche de base (SB) sont plutôt caractérisées par un indicateur de prévision normal.

Cette performance recouvre la mise au point d'un plan de formation $(2,42)$, d'un plan prévisionnel des ventes $(1,70)$, de l'analyse de la rentabilité prévisionnelle des investissements $(1,35)$ et de l'établissement d'un plan prévisionnel de production $(1,30)$.

Le suivi porte essentiellement sur la production et concerne le suivi mensuel du stock des matières $(2,49)$ et du nombre d'heures travaillées par rapport aux heures ouvrées $(1,94)$. Les délais clientèle ne requièrent pas un suivi quotidien $(-1,97)$, mais plutôt mensuel $(1,57)$. La trésorerie est examinée chaque jour $(1,56)$ tandis que la marge commerciale l'est chaque mois $(1,56)$. Enfin, l'ensemble du contrôle de gestion est effectué par un service spécialisé de l'entreprise $(2,71)$.

Les résultats qui viennent d'être présentés méritent au moins deux commentaires.

Le premier concerne le «score» obtenu par les PMI SI, en matière d'indicateur de suivi, relativement au reste de la population examinée. Pour surprenant qu'il soit, cet indicateur de niveau très faible renvoie à la structuration du modèle de suivi qui, dans ce type de PMI, est la plus lourde à mettre en œuvre. Pour ces firmes, la production est en effet organisée en technologies homogènes (sections homogènes) afin de profiter au mieux des économies d'échelle. Dans ces conditions, le suivi d'une commande, tel qu'il est spécifié dans notre questionnaire, est alors plus difficile à effectuer, car le suivi correspond plus à une logique de rentabilité de chaque section, considérée comme un centre de responsabilité. 
Le deuxième commentaire concerne l'opposition qui, du point de vue de l'indicateur de prévision, permet de séparer nettement les PMI $S B$ et $S I$, qui développent une prévision normale, des PMI $S S$, pour lesquelles l'indicateur est très inférieur. Cette séparation résulte en partie seulement d'un effet taille. Les résultats statistiques montrent, d'une part, que la croissance de la taille, exprimée en termes d'effectif salarié, est positivement corrélée avec le degré de développement des outils de prévision et, d'autre part, que les PMI SS sont de taille sensiblement inférieure à celle des PMI SI et SB ${ }^{9}$. Pourtant, l'effet taille n'explique pas l'intégralité de l'opposition observée, ne serait-ce que parce que la classe des PMI SS comporte également des firmes de grande taille ${ }^{10}$. En fait, cette opposition renvoie également à la délimitation du cœur de la firme relativement à son espace concurrentiel. Le premier élément d'explication concerne l'autonomie de l'entreprise. Les firmes autonomes (qui constituent $54 \%$ des $S S$ ) sont en effet caractérisées par un indicateur de prévision inférieur $(1,32)$ et très inférieur $(2,08)$, exclusif de normal $(-2,96)$, tandis que les filiales ( $8 \%$ des $S S, 50 \%$ des $S I$ et des $S B$ ) et les établissements le sont par un indicateur de prévision normal $(2,37$ pour les premières et 1,31 pour les seconds) ${ }^{11}$. Le second élément d'explication concerne l'insertion de la firme dans un réseau de sous-traitance. Ainsi, le traitement statistique des données recueillies montre que l'insertion de la PME dans une relation de soustraitance dégrade les capacités d'analyse et de prévision, par rapport aux entreprises non sous-traitantes. En outre, la croissance de la part de la soustraitance dans le CA accroît le degré de développement des outils d'analyse de l'activité et amoindrit celui de ses outils de prévision ${ }^{12}$. Or, les PMI $S I$ et $S B$ de notre échantillon effectuent très peu de sous-traitance $(2 \%$ du CA en moyenne), tandis que les relations avec des donneurs d'ordres représentent environ $12 \%$ du CA des $S S$.

9. L'effectif salarié moyen est de 260 pour les traditionnelles, de 579 pour les scale intensive, de 82 pour les specialized suppliers et de 727 pour les science based.

10. En outre et si l'on exprime la taille de la firme en fonction du chiffre d'affaires, l'idée d'un effet taille exclusif n'est pas plus convaincante. En effet, les CA moyens sont de $210 \mathrm{MF}$ pour les traditionnelles, de 1706 pour les scale intensive, de 58 pour les specialized suppliers et de 853 pour les science based. Dans ces conditions, si l'effet taille peut expliquer l'opposition specialized suppliers / scale intensive, science based, il ne permet cependant pas de rendre compte de l'homogénéité du scoring de ces derniers en matière de prévision, alors que les scale intensive réalisent un CA moyen équivalent au double de celui des science based. $S$ 'il existe un effet seuil, celui-ci ne relève alors pas, par hypothèse, du critère chiffre d' affaires.

11. Sur ce point voir Fernandez, Picory et Rowe (1993).

12. Ibidem. 


\section{Espaces concurrentiels et métamodèles de contrôle}

Les commentaires qui précèdent évoquent la possibilité d'une caractérisation des problématiques de contrôle de gestion ${ }^{13}$ des firmes en fonction des propriétés des espaces concurrentiels dans lesquels elles s'inscrivent. Par concept d'espace concurrentiel, nous entendons, de manière générale, l'ensemble des variables essentielles qui déterminent la dynamique des firmes sur les marchés. Ce concept recouvre a priori les choix technologiques, l'échelle et les techniques de la production, les conditions réglementaires de celle-ci, l'insertion dans des rapports de sous-traitance, les conditions de la demande (marchés publics, exportation, etc.) ainsi que les contraintes de marché au sens classique (prix, concurrence, etc.).

À cet égard et du point de vue de la problématique du contrôle de gestion, l'étude effectuée permettrait de distinguer deux types distincts de contrôle de gestion propres aux espaces concurrentiels qui correspondraient à deux métamodèles de contrôle : un modèle dont la clé de voûte serait la planification et un autre structuré autour du pilotage.

Le premier est celui des firmes caractérisées par une dimension organisationnelle élevée (SI) et des firmes orientées par une forte intensité de recherche de base $(S B)$; il correspondrait à un modèle de régulation ex ante des activités de la firme.

De taille relativement importante, les firmes $S I$ sont très exposées à la concurrence, nationale et internationale. Cette concurrence porte d'une part sur les facteurs prix : sensibilité sur les prix de vente, mais aussi sur les coûts de production et, d'autre part, sur les facteurs hors prix : performance des réseaux de distribution, variété de gammes, qualité, fiabilité, publicité, mais aussi capacités à intégrer les contraintes réglementaires de toutes natures, etc. Cette spécificité concurrentielle est liée à l'échelle importante de la production. Toutefois, cette spécificité fondamentale n'exclut nullement l'innovation; celle-ci s'inscrirait plus dans le cadre d'une stratégie de réduction des coûts. Pour ces entreprises, l'innovation radicale n'est pas, elle non plus, exclue. Elle implique cependant une bonne compatibilité entre les contraintes de la production sur grande échelle et le risque lié à l'innovation radicale, en particulier, celui d'un mauvais retour sur investissement en R-D, si le produit innovant est affecté d'un cycle de vie très court. Une des solutions à ces difficultés consiste à «sous-traiter» le risque auprès de petites PME $S B$. Il s'instaure alors entre ces deux types de firmes une complémentarité, les unes chargées de mettre au

13. Dans leur dimension instrumentale. 
point un nouveau paradigme technique, les autres plus spécialisées dans l'exploitation commerciale générale et la diffusion rapide des produits innovants sur les marchés. Dans cet esprit, l'existence de PME innovantes ne renvoie pas seulement à des occasions favorables pour les grandes firmes, mais témoigne aussi de la nécessité de structures adaptées et de petites dimensions pour la prise en charge de certaines fonctions industrielles.

Des développements qui précèdent il résulte que la dynamique des firmes $S I$ est déterminée à la fois par des contraintes de nature technologique et par des contraintes concurrentielles classiques au titre desquelles il faut compter non seulement les facteurs de compétitivité prix, les capacités à innover dans le cadre d'une stratégie de réduction des coûts, mais encore des facteurs plus diffus tels que la performance des réseaux de distribution, etc.

De taille comparable aux précédentes, les firmes $S B$ maîtrisent relativement bien leur « univers » concurrentiel, ce qui ne signifie pas qu'elles soient abritées de la concurrence. Cette maîtrise renvoie en partie à une spécialisation technique qui correspond à une dynamique innovante proprement interne. Les comportements innovants induisent alors une bonne autonomie des unités de production, y compris lorsque leur activité s'inscrit dans des relations de sous-traitance.

Une autre caractéristique importante de ce type d'entreprises peut être trouvée dans leur insensibilité relative aux facteurs de compétitivité prix, qu'il s'agisse du prix, des outputs ou de celui des inputs. Pour ces PME, la concurrence porte essentiellement sur des facteurs hors prix : avantage technologique, savoir-faire, compétitivité du bureau d'étude.

Les deux catégories semblent correspondre à un métamodèle de contrôle structuré par une «boucle» procédurale : la planification-pilotage. La clé de voûte du modèle de contrôle est la planification: la régulation, si ce n'est la rationalisation, des activités se fait ex ante; le pilotage permet de «boucler» sur celle-ci. La fréquence de suivi des indicateurs de pilotage est relativement faible et sert à réagir vis-à-vis des prévisions dans une logique d'apprentissage organisationnel pour certains indicateurs et pour d'autres, de façon habituelle.

La cohérence interne du modèle des firmes $S B$ semble par ailleurs plus forte que celle des firmes $S I$ : la relative exhaustivité des indicateurs de suivi des premières couvre l'ensemble des dimensions du contrôle déployées par le processus de planification.

La seconde catégorie est celle des firmes orientées par la demande (SS) et traditionnelles (ST); elle correspond à un modèle de contrôle ex post (ou in-situ) des activités de la firme. 
De taille plus restreinte par rapport au groupe précédent, les PME SS maîtrisent relativement bien leur « univers » concurrentiel, à l'image des firmes $S B$. Cette maîtrise correspond à une spécialisation technique qui renvoie à une dynamique innovante «tirée par la demande». La différence entre ces deux types d'entreprises ( $S B$ et $S S$ ) relève du rôle que joue l'innovation dans la structuration: pour les unes $(S B)$, l'innovation peut être qualifiée de technology push, c'est-à-dire dont la dynamique est liée en majeure partie à celle de la recherche fondamentale et appliquée; pour les autres (SS ), l'innovation peut être qualifiée de demand pull, c'est-à-dire dont la dynamique est liée à celle de la demande. Cette différenciation conduit naturellement à deux profils de développement des firmes bien distincts : le premier est davantage lié à la performance du bureau d'étude et à celle des recherches concurrentes, si elles existent $(S B)$; le second est plus lié aux capacités de s'adapter aux exigences particulières de la clientèle ( $S S$ ) sur le plan non seulement technique, mais aussi économique, en particulier en matière de coûts. Pour ces dernières, la concurrence porte donc essentiellement sur des facteurs hors prix : flexibilité, fiabilité, qualité des produits, capacités d'adaptation et d'assistance.

De taille sensiblement équivalente à celle des entreprises du groupe précédent, les PME traditionnelles s'inscrivent dans un contexte concurrentiel plus classique. Celui-ci est en effet caractérisé par une forte concurrence sur les prix, ainsi que par une volonté de protection par rapport aux risques et aux incertitudes liés à celle-ci. Cette volonté est concrétisée par l'inscription des firmes dans des relations de sous-traitance, par le soumissionnement au marché public et par une faible ouverture sur l'extérieur.

La contrepartie de la création de cette sphère de protection peut être trouvée, d'une part, dans la perte relative d'autonomie en matière de choix techniques et de fixation des prix et, d'autre part, dans l'absence d'activité innovante, faiblement stimulée par la concurrence. Cette idée doit cependant être nuancée en raison du caractère traditionnel de la production pour laquelle l'avantage concurrentiel correspond plus, soit à une stratégie de réduction des coûts, en vertu de la grande sensibilité aux prix des inputs, soit à des efforts consentis pour l'amélioration de la commercialisation des produits.

La dynamique de ces firmes est très sensiblement dépendante non seulement des paramètres classiques tels que le prix de vente et les coûts des facteurs, mais encore, et à titre principal pour les PME de cette classe, des composantes de la demande, en termes de sous-traitance et de marchés publics.

Ces deux catégories semblent correspondre à un métamodèle de contrôle structuré par une logique de "pilotage à vue», avec là encore une différence (hiérarchie ?) dans les niveaux de cohérence interne des systèmes de contrôle de chacune des deux catégories représentatives de ce métamodèle. 
Rappelons que les PME des secteurs SS sont caractérisées par un indicateur de prévision très inférieur. Il est vrai que l'argument suivant lequel les entreprises qui évoluent dans cet espace concurrentiel peuvent difficilement élaborer une planification opérationnelle est presque un truisme ${ }^{14}$. Le pilotage se fait à partir des «affaires» (essentiellement par le suivi unitaire du coût de fabrication). En revanche, les indicateurs de suivi permettant la rationalisation ex post sont peu nombreux (corrélation négative avec le suivi des taux d'utilisation des équipements, marge commerciale suivie trimestriellement). On peut alors supposer que le pilotage sert la régulation de l'activité et non sa rationalisation ${ }^{15}$.

Les PME des secteurs traditionnels, si elles s'inscrivent dans cette problématique du contrôle circonscrit à celle du pilotage, s'appuient sur un modèle plus dense sur le plan de la cohérence interne. Les outils de suivi sont supérieurement développés et les outils d'analyse normalement développés; or, ces derniers servent notamment à «normer» les premiers.

L'opposition entre les deux modèles de contrôle est liée à la manière dont les firmes perçoivent l'incertitude et tentent de la limiter.

Dans le cadre du premier modèle, la prévision correspond à des anticipations de la dynamique des formes de la concurrence et de la technologie. À ces anticipations est associée une planification correspondant à des stratégies dans lesquelles les entrepreneurs, non seulement organisent les processus de production, assurent l'interface entre science et marché par l'intermédiaire des innovations de procédés et de produits, mais encore restructurent les marchés au moyen d'alliances, de fusions et d'acquisitions, d'accords de coopération, en particulier en matière de R-D (Eliasson, 1986; De Jong, 1990 ; Schumpeter, 1912 et 1939). Ces stratégies d'entreprises supposent alors un positionnement à long terme et impliquent une limitation de l'incertitude au sens fort, au moyen d'un contrôle proactif des relations de la firme avec son environnement. Cela signifie que non seulement les entreprises se projettent dans l'avenir, mais aussi qu'elles innovent suffisamment pour coproduire leur environnement.

Dans le cadre du second modèle, la prévision correspond plus à une procédure d'ajustement à l'incertitude radicale en matière de positionnement

14. Sauf, il est vrai, par des stratégies de «partenariat », de fidélisation de la clientèle ou d'études de marché... qui sont autant d'éléments qui s'inscrivent plutôt dans la planification stratégique.

15. On peut, sans être normatif, se poser la question de la pertinence des coûts calculés puisque les indicateurs qui permettent d'optimiser la détermination des taux horaires ne sont pas suivis. 
de la firme sur son marché et de dynamique concurrentielle. Comme dans le cas précédent, les instruments de planification restent liés à la manière dont la firme perçoit l'incertitude. Elle tente de la limiter au moyen d'un contrôle non pas proactif, mais plus adaptatif, des variables essentielles d'un contexte concurrentiel plus classique, ou encore en se ménageant des «niches» concurrentielles, liées à leur flexibilité et leurs capacités d'adaptation et d'assistance, à la qualité et à la fiabilité de leurs produits.

\section{Conclusion}

Dans le cadre d'une étude antérieure (Fernandez, Picory et Rowe, 1993), l'approche contingente du développement des outils de gestion a confirmé l'importance de l'effet taille de l'entreprise. Mais l'analyse de l'influence des facteurs économiques classiques, comme l'appartenance sectorielle, n'apportait pas d'explication structurelle satisfaisante à cette corrélation. A contrario et dans le cadre de cette étude, le positionnement des entreprises dans leur espace concurrentiel respectif permet de nuancer, voire d'atténuer en grande partie cet effet. Ce dernier n'apparaît que comme une conséquence d'une structuration plus fondamentale approchée par la typologie présentée. De plus, cet effet ne joue plus dans aucun des secteurs au sens de Pavitt (1984). Au sein d'un même secteur, en effet, la nature des outils de contrôle apparaît très fortement liée aux caractéristiques des technologies mises en œuvre et à celles de l'offre et de la demande.

Cette explication de l'effet taille par la typologie des espaces concurrentiels a d'importantes implications. En premier lieu, l'absence apparente de certains outils de gestion n'est pas rédhibitoire en soi et la croissance de l'entreprise ne peut y remédier que si la taille de l'entreprise est effectivement sous-optimale, par rapport à l'espace concurrentiel dans lequel elle s'inscrit. En second lieu, l'orientation du contrôle ex post ou ex ante, qui nous a permis de discriminer deux «métamodèles » de contrôle de gestion, paraît liée à la conjonction de variables relevant à la fois de l'espace production et de l'espace marché; conjonction que nous avons qualifiée d'espace concurrentiel. Cela permet de confirmer l'hypothèse selon laquelle l'analyse de la coordination et de l'organisation des activités de production s'inscrit dans une dialectique «firme-contexte de l'activité » et cela en dépit du caractère extrêmement fluctuant de la frontière de la firme.

Pour autant, l'économique ne détermine pas exclusivement l'incitation à développer tel ou tel outil. Le comportement de l'entrepreneur et la stratégie de la PME jouent certainement un rôle dans la structuration et le développement de ces outils (Marchesnay, 1991). Cependant, le rôle du dirigeant, dans la définition des outils de gestion, pourrait résulter principalement d'une 
stratégie d'adaptation à son espace concurrentiel. Est-ce à dire que ces résultats et interprétations limitent le dirigeant à cette fonction d'adaptation? Le prétendre serait certainement méconnaître le rôle central qu'il joue en tant que manager (Lebraty, 1992) dans les décisions qui scellent le destin de l'entreprise.

\section{Annexes}

\section{Les caractéristiques du processus de production}

\section{Annexe 1}

La logique de la question A.13. du questionnaire était d'amener les chefs d'entreprises à caractériser les contraintes essentielles qui pèsent sur l'activité de production et sur la dynamique de leur firme et cela à partir d'un nombre limité de critères qui n'étaient pas uniquement d'ordre technologique, ni totalement exclusifs.

Ces critères étaient les suivants :

- production standardisée;

- production sur grande échelle (grandes séries);

- production contrainte par les économies d'échelle;

- production contrainte par les facteurs de compétitivité prix;

- production caractérisée par une gamme étendue de produits;

- production caractérisée par sa haute différenciation en fonction des exigences de la clientèle ;

- production déterminée par la qualité de la recherche fondamentale ou appliquée ;

- production caractérisée par un haut contenu technologique (brevets, etc.);

- production contrainte par les efforts en matière de présentation des produits, de publicité, d'emballage, de marques.

Cette liste de caractéristiques mérite quelques commentaires.

Tout d'abord, la production sur grande échelle recouvre, d'une part, des volumes élevés de production et, d'autre part, des immobilisations, consommations intermédiaires et effectifs salariés importants. Implicitement à ces caractéristiques de production est également prise en compte la taille du marché, quelle que soit la localisation géographique de ce dernier.

Le second commentaire concerne la recherche et les comportements d'innovation. En général, recherche et innovation sont appréciées quantitativement; on utilise alors des indicateurs comme le budget consacré à la 
recherche et au développement (R-D), le nombre de chercheurs et d'ingénieurs dans l'effectif salarié, voire le nombre de brevets déposés. Si nous utilisons ces critères et d'autres encore, il est classique aujourd' hui d'observer une diffusion lente de l'innovation technologique dans le tissu des PMI.

Les études montrent en effet que les efforts consacrés à la R-D par les grandes entreprises représentent un peu plus de $5 \%$ de leur CA, et que ces dépenses diminuent très rapidement avec la taille de l'entreprise : en pourcentage du CA, elles ne représentent plus que $0,4 \%$ pour les PMI de 50 à 100 salariés et sont quasiment nulles pour les PMI de plus petite taille. En 1989, le CEPME estimait que sur l'ensemble des quelque 30000 PMI françaises employant 20 à 499 salariés, 1250 seulement disposent de l'équivalent d'un chercheur à plein temps. Par ailleurs, d'après le ministère de l'Industrie, 2000 PMI seulement ont demandé à bénéficier du crédit d'impôt-recherche ${ }^{16}$. L'étude du CEPME de 1986 révèle que $28 \%$ des PMI de 10 à 499 salariés ont réalisé des dépenses pour l'innovation technologique (produits, outils ou procédés de fabrication), alors que $15 \%$ auraient engagé des dépenses de stylisme industriel (maquettisme et design). Ces chiffres représentent un net progrès sur les niveaux enregistrés en 1980. Pour cette année, en effet, $20 \%$ des PMI avaient engagé des dépenses de recherche et $10 \%$ seulement des frais pour le stylisme industriel.

Les conceptions restrictives de l'innovation et de la recherche, corrélatives aux instruments de mesure utilisés, conduisent à singulariser négativement les PMI dans le mouvement général de changement technique au sens large: les PMI innoveraient et rechercheraient peu.

Nous considérons cependant que, bien qu'il faille prendre en compte des indicateurs comme les budgets R-D, le nombre de brevets, les effectifs chercheurs, les ingénieurs, etc., il ne convient pas de se limiter à ces seuls indicateurs, sous peine de ne voir dans la PME qu'une grande entreprise en devenir : ces indicateurs n'ont en effet de sens que pour les grandes entreprises, seules en mesure de mobiliser des ressources financières et humaines en R-D. Cela dit, le fait que les PMI n'atteignent pas le seuil «statistique» des $5 \%$ du CA en R-D n'implique pas leur exclusion du processus général de renouvellement de la technologie et de la recherche industrielle ${ }^{17}$.

En outre, et de manière plus générale, ces indicateurs véhiculent une conception techniciste de l'innovation. Si effectivement le changement

16. Ces chiffres sont à comparer à ceux de la RFA où 20000 entreprises, dont certaines très petites, ont reçu la prime d'aide aux salaires des personnels affectés à la R-D. Le crédit d'impôt français est une mesure comparable.

17. Pour des indications très générales sur la contribution des PME au changement technique, on pourra consulter Callon, 1986. 
technique ${ }^{18}$ peut dans certains cas constituer l'aboutissement d'un plan de recherche interne à l'entreprise et pour lequel on mobilise effectivement un certain nombre de moyens objectivement quantifiables, il peut également renvoyer à une diffusion de l'innovation par le biais de l'investissement ou au moyen d'une synergie entre les centres de recherche-enseignement publics et l'industrie, voire à un processus plus subtil et qualitatif d'apprentissageperfectionnement ou encore, à un savoir-faire correspondant à la spécialité de l'entreprise. Il faut donc opter pour une conception plus qualitative du changement technique (produit ou procédé), qu'il soit strictement interne à la firme ou encore validé par le marché (innovation économique au sens propre du terme).

\section{Nomenclature sectorielle (Pavitt [1984]) par rapport à la nomenclature SITC}

\begin{tabular}{|c|c|c|}
\hline $1-$ & SECTEUR «TRADITIONNEL» & 2 - SECTEUR «SCALE INTENSIVE» \\
\hline & $\begin{array}{l}\text { Bijouterie } \\
\text { Chaussures } \\
\text { Articles de voyage } \\
\text { Mobilier } \\
\text { Habillement } \\
\text { Minerais non métallurgiques } \\
\quad \text { (céramique) } \\
\text { Textiles } \\
\text { Produits métalliques } \\
\text { Laine - Bois } \\
\text { Jeux et articles de sports } \\
\text { Peaux et Cuirs } \\
\text { Instruments musicaux } \\
\text { Verre } \\
\text { Autres }\end{array}$ & $\begin{array}{l}\text { Électroménager } \\
\text { Constructions navales } \\
\text { Pneumatiques } \\
\text { Sidérurgie } \\
\text { Chimie non organique } \\
\text { Fibre } \\
\text { Automobile } \\
\text { Composants électriques } \\
\text { Papier } \\
\text { Matières plastiques } \\
\text { Vernis - Peintures } \\
\text { Chimie organique } \\
\text { Radio T.V. } \\
\text { Engrais } \\
\text { Métaux non ferreux } \\
\text { Horlogerie }\end{array}$ \\
\hline $3-$ & $\begin{array}{l}\text { SECTEUR } \\
\text { «SPECIALIZED SUPPLIERS» }\end{array}$ & 4- SECTẸUR «SCIENCE BASED» \\
\hline & $\begin{array}{l}\text { Machines pour l'industrie alimentaire } \\
\text { Imprimerie - Édition } \\
\text { Machines agricoles } \\
\text { Machines outils } \\
\text { Véhicules ferroviaires } \\
\text { Machines électriques } \\
\text { Moteurs électriques } \\
\text { Machines extractives } \\
\text { Instrumentation médicale } \\
\quad \text { de mesure et de contrôle } \\
\text { Machines de bureau } \\
\text { Audio - Vidéo } \\
\text { Autres machines }\end{array}$ & $\begin{array}{l}\text { Aéronautique } \\
\text { Moteurs pour aéronautique } \\
\text { Pharmacie } \\
\text { Antiparasite } \\
\text { Instruments de précision électroniques } \\
\text { Informatique } \\
\text { Armement } \\
\text { Électronique médicale } \\
\text { Télécommunications } \\
\text { Chimie fine } \\
\text { Cosmétique, hygiène, parfumerie } \\
\text { Réacteurs nucléaires }\end{array}$ \\
\hline
\end{tabular}

18. Qu'il s'agisse d'un changement concernant les techniques de production (innovation procédé) ou encore les produits (innovation produit). 


\section{Représentativité de l'échantillon}

\section{Annexe 2}

Comparaison entre les statistiques du SESSI (1990/1991), l'échantillon de la Centrale des Bilans de la Banque de France et l'échantillon constitué pour la présente étude (PME industrielles).

\begin{tabular}{lccc}
\hline Établissements & $<\mathbf{5 0}$ salariés & Entre $\mathbf{5 0}$ et $\mathbf{5 0 0}$ salariés & Total \\
\hline SESSI & & & \\
Île-de-France & $\mathbf{6 3 4 6}(\mathbf{7 6 , 9} \%)$ & $\mathbf{1 9 0 1}(\mathbf{2 3 , 1} \%)$ & $\mathbf{8 2 4 7}(\mathbf{1 0 0} \%)$ \\
Autres régions & 16939 & 8999 & 25938 \\
Ensemble & 23285 & 10900 & 34184 \\
Île-de-France & $27,20 \%$ & $17,40 \%$ & $24,10 \%$ \\
Autres régions & $72,30 \%$ & $82,60 \%$ & $75,90 \%$ \\
Ensemble & $100 \%$ & $100 \%$ & $100 \%$ \\
\hline
\end{tabular}

\section{Échantillon}

Centrale des

Bilans

\begin{tabular}{lccc}
$\begin{array}{l}\text { Île-de-France } \\
\text { Échantillon }\end{array}$ & $\mathbf{2 4 8}(\mathbf{5 8 , 1} \%)$ & $\mathbf{1 7 9}(\mathbf{4 1 , 9} \%)$ & $\mathbf{4 2 7}(\mathbf{1 0 0} \%)$ \\
$\begin{array}{l}\text { ENST } \\
\text { Île-de-France }\end{array}$ & $70(64,3 \%)$ & $39(35,7 \%)$ & $109(100 \%)$ \\
\hline
\end{tabular}

\section{Bibliographie}

Anthony, R.N. (1988), The Management Control Function, Cambridge, Harvard Business School Press, p. 216.

CALlON, M. (1986), «La recherche industrielle: le mal français », La Recherche, Supplément Économie, ${ }^{\circ} 183$, p. 4-10.

COASE, R.H. (1937), « The nature of the firm », Economica, no 4, p. 386-405.

D'AmBoise, G. et A. BAKANiBona (1990), «La planification dans les PME: une synthèse de résultats empiriques. Conclusions et recommandations », Revue Internationale PME, vol. $3, \mathrm{n}^{0}$ 2, p. 147-166.

DE JONG, H.W. (1990), «On market theory », dans B. Dankbaar, J. Groenenwegen et H. Schenk (éd.), Perspectives in Industrial Organization, Dordrecht, Kluwer, p. $29-40$.

Dosi, G., K. PavitT et L. Soete (1985), The Economics of Innovation and International Trade, Brighton, Wheatsheaf. 
Eliasson, G. (1986), «International competition, productivity change and the organization of production », dans H.W. De Jong et W.G. Shepherd (éd.), Mainstreams in Industrial Organization, Dordrecht, Kluwer, p. 127-158.

FernandeZ, V., C. PiCORY et F. Rowe (1993), Les outils de gestion dans les PME, Livre Blanc, Paris, Département économie et management, ENST, p. 94.

FERNANDEZ, V., C. PICORY et F. Rowe (1994), «Diversité, cohérence et pertinence des outils de gestion: le cas des PME d'Île-de-France », Communication présentée à la $39^{\mathrm{e}}$ International Council on Small Business World Conference, Strasbourg, 27-29 juin 1994, p. 295-304.

Lebraty, J. (1992), «Management et gestion : quel apprentissage ?», Économie et Société, Série Sciences de gestion, $\mathrm{n}^{0}$ 18, p. 131-159.

MARChESNAY, M. (1991), «La PME: une gestion spécifique? », Économie Rurale, novembre-décembre, réédité dans Problèmes Économiques, nº 2276, 20 mai 1992, p. 26-32.

PAVITT, K. (1984), «Sectoral patterns of technical change : towards a taxonomy and a theory », Research Policy, n 13, p. 343-373.

PICORY, CH. (1990a), Prix et environnement concurrentiel des PME, Paris, ENST, Département économie et management, p. 135.

PICORY, CH. (1990b), «PME, comportements d'innovation et espaces concurrentiels », Communication au colloque La PME: objet de recherche pertinent ?, TETRAUniversité de Lyon II, 30 et 31 mai, p. 32.

Robinson, R.B. et J.A. PEARCE (1984), «Research thrusts in small firm strategic planning », Academy of Management Review, vol. 9, n 1, p. 128-137.

SCHUmaCher, E.F. (1973), Small is beautiful. Traduction française, Paris, Seuil.

SCHUMPETER, J.A. (1912), The Theory of Economic Development, $4^{\mathrm{e}}$ édition, Cambridge, Harvard University Press. Traduction française, 1935, Paris, Dalloz.

SCHUMPETER, J.A. (1939), Business Cycles, New York, McGraw-Hill.

SCHUMPETER, J.A. (1942), Capitalism, Socialism and Democracy, Londres, Allen et Unwin. Traduction française, Payot, 1990, p. 451. 